\title{
LA TRADUCTION EN POLONAIS DES TEXTES D'ANCIEN FRANÇAIS: UNE COMPÉTENCE SPÉCIFIQUE
}

\begin{abstract}
Giermak-Zielinska Teresa, La traduction en polonais des textes d'ancien français: une compétence spécifique [The translation of ancient French texts into Polish: a specific translator's competence]. Studia Romanica Posnaniensia, Adam Mickiewicz University Press, Poznań, vol. XXX: 2003, pp. 73-81. ISBN 83-232-1270-8. ISSN 0137-2475.

This paper is relating a practical experience of teaching Romance philology students the translation from ancient French into Polish. The main scope is a restitution of an ancient text respecting not only the equivalence at the lexical and syntactical level, but also the discourse structures, such as the linear sequence of events and events related from different points of view: some cxamples of solving particular problems arc discussed. The whole procedure resembles that of Iranslating from Latin, rather than a translation from one modern language to another.
\end{abstract}

Les quelques réflexions qui vont suivre sont le fruit d'une expérience purement didactique: depuis quelques années je dirige un séminaire de traduction, consacré essentiellement aux textes français des $\mathrm{XI}^{\mathrm{e}}-\mathrm{XIII^{ \textrm {e } }}$ siècles. Le choix du séminaire est optionnel, la matière n'est pas des plus faciles; cependant, le séminaire réunit tous les ans un groupe d'étudiants intéressés par ce sujet. Afin de rendre le contact avec le texte ancien plus immédiat, je n'emploie que des textes jamais encore traduits en polonais tout en conseillant d'éviter, à la première approche du texte, la consultation des traductions françaises modernes. Ces traductions ne seront employées que plus tard, dans le but d'enrichir la réflexion sur les moyens langagiers disponibles et l'usage dont le traducteur en fait.

Demandés à l'ouverture du séminaire de motiver leur choix, les étudiants répondent qu'ils espèrent acquérir un certain savoir-faire profitable pour une future carrière de traducteur, encore qu'ils se rendent compte que seulement peu d'élus auront la chance de faire connaître leur nom au grand public. Afin de répondre à ces attentes, j'essaie de mettre en œuvre un certain nombre de postulats pratiques, en espérant qu'ils seront utiles pour d'autres textes, au-delà des exercices de traduction de l'ancien français vers le polonais. 
Tout enseignement pratique de la traduction comporte un enjeu commun: il s'agit de sensibiliser l'étudiant aux différences des deux systèmes linguistiques. En ce qui concerne l'ancien français, l'étudiant aura vite compris que la compétence langagière dans ce domạine diffère sensiblement de celle du français moderne. Tandis que pour les textes français contemporains l'étudiant dispose d'une connaissance active de la langue de départ, la traduction de l'ancien français serait plutôt comparable à la version latine. Dans celle-ci, comme dans celle-là, le décodage du texte nécessite un travail plus raisonné que spontané, visant à déterminer les fonctions des constituants et les relations entre eux, afin de reproduire le même ordre d'idées dans la traduction. En observant le travail de l'étudiant sur un texte ancien on constate que, la plupart du temps, il ne se rend pas compte de la différence systémique entre l'ancien français et le français moderne; en d'autres termes, que l'ancien français est doté d'un système grammatical autonome.

Une deuxième observation accompagne la précédente: étant donné la relative complexité du texte ancien, l'étudiant se concentre presqu'uniquement sur la compréhension de celui-ci, se souciant très peu de la forme que prendra sa traduction. Une fois la restitution du sens terminée, il considère son travail fini. Cela est vrai même des étudiants doués d'une excellente connaissance du français et d'une bonne base grammaticale pour l'ancien français. D'où vient donc cette déficience? Je pense que la principale raison en est que les étudiants ont trop peu l'habitude de réfléchir sur l'usage de leur langue maternelle. Le savoir scolaire dans ce domaine est, me semble-t-il, de loin insuffisant. Or, l'utilisation consciente et réfléchie de la langue maternelle devrait reposer sur des connaissances métalangagières telles que la grammaire, la stylistique, enfin certaines notions de base concernant le fonctionnement du langage en général. $\mathrm{Si}$, à la liste des déficiences scolaires, on ajoute la place des langues classiques réduite quasiment à zéro, ainsi que l'application d'une méthode uniquement communicative pour les langues vivantes, on constatera que l'étudiant prend l'habitude à réfléchir sur les mécanismes linguistiques seulement à l'université.

Un étudiant en langues vivantes et lettres modernes, en tant qu'un futur professeur de langues ou traducteur / interprète, devrait donc compléter son apprentissage par quelques rudiments concernant le fonctionnement du langage en général. Je ne prétends pas en faire un linguiste, mais il devrait compter des linguistes parmi ses maîtres. Il n'est pas forcé de faire une recherche linguistique sur le texte qu'il traduit, mais il doit faire siens des outils d'analyse qui lui permettront de répérer les difficultés et trouver des solutions adéquates. En clair donc: l'étudiant doit posséder des compétences métalangagières d'analyse, basées sur des procédures linguistiques, mais orientées vers un but pratique.

Ces compétences métalangagières d'analyse sont centrées, comme on sait, sur trois objectifs:

- saisir les significations linguistiques des unités du texte de départ, afin de pouvoir ensuite; 
- comprendre, à travers une globalisation des résultats partiels, le sens du message, avec toutes les connotations qu'il véhicule;

- restituer le message en termes d'équivalence de la langue d'arrivée.

Un décodage complet consiste donc en une démarche analytique consciente pour laquelle un futur traducteur devrait être muni d'outils adaptés à ses besoins. Par exemple, en ce qui concerne l'organisation des unités du texte, l'outil qui ferait probablement l'affaire serait une analyse «logique» rénovée et enrichie de la notion de cohérence textuelle, en particulier en ce qui concerne la succession narrative des événements, les arguments et les conclusions. Mais attention: les mêmes procédures analytiques devraient être appliquées au texte source et au texte cible, ce qui présuppose une connaissance égale des deux systèmes linguistiques.

La restitution du message en termes de la langue d'arrivée comporte des embûches bien plus graves, car c'est là où le traducteur doit faire des choix, souvent difficiles, compte tenu des réalités culturelles et historiques différentes. Un tel choix n'est, comme on sait, jamais tranchant, surtout en ce qui concerne un texte qui se réfère à une réalité très éloignée de celle connue par le traducteur et le lecteur présumé. Sans m'engager dans l'éternel débat sur le degré de fidélité de la traduction à l'original ancien, je propose aux étudiants la réfléxion sur des problèmes ponctuels, mais importants pour bien saisir le contenu du texte. Par exemple, on suivra d'assez près l'organisation de l'énoncé et l'agencement du récit en essayant de déterminer par quels moyens grammaticaux / lexicaux est exprimée la progression des événements et comment s'opère la transition entre le déroulement linéaire de l'action et des événements rapportés dans la narration. Ici, la recherche des points de vue (des énonciateurs) différents offre un précieux outil d'analyse. Quant au niveau lexical, la réflexion est ciblée sur des difficultés ponctuelles, propres à un texte donné; par exemple sur une terminologie ancienne à découvrir, sur la question d'équivalence des noms propres et des toponymes, etc.

Voici donc quelques exemples de problèmes pratiques auxquels les étudiants du séminaire sont priés de rétléchir.

\section{LA PROGRESSION DU RÉCIT}

L'une des tâches du traducteur est de sauvegarder la progression du récit, car c'est là où le principe de la fidélité doit être respecté sans concession. Le principal moyen ici est de savoir approprier les formes verbales et les circonstants à la séquence des événements.

En ancien français le récit se fait de manière altemée: le "plan du passé» recoupe le "plan du présent» souvent dans le cadre d'une même phrase complexe. On a l'impression que le «plan du passé» constitue dans de tels cas une toile de fond, une mise en scène qui prépare l'auditeur à se concentrer pour suivre le 
déroulement de l'histoire, représenté par un /des repère(s) actualisant(s) que j'appellerai volontiers le «moment présent textuel»:

Exemple 1. (Renart, branche 1, 266-271) ${ }^{1}$

Quant Ysengrin ö le roi

Qui de la pes prenoit conroi,

Molt fu dolanz, ne set que faire ${ }^{2}$

Ne n'en set mes a quel chef trere.

Sur la terre, entre deus eschames,

S'asiet la coue entre les janbes.

Traduction: En entendant le roi / se faire le défenseur de la paix, / Isengrin fut affligé, il ne sait que faire / ni quel parti prendre. / Il s'assied entre deux tabourets à même le sol, / la queue entre ses jambes.

La traduction en français moderne suit, dans une certaine mesure, le schéma temporel de l'ancien français en évitant cependant, par l'emploi de la participiale et l'infinitive (en italique dans le texte), un voisinage trop marqué du présent et du passé simple. Le passage au présent marque un développement dynamique du récit.

Exemple 2 (Renart, branche 1, 345-350)

Quant revindrent de paumoisons

si con nos en escrit trovons ${ }^{3}$

La ou lo roi virent seoir

Totes li vont au pié chaoir,

Et Chantecler si s'ajenoille

Et de ses lermes ses piez moille.

Traduction: Revenues à elles, / comme nous dit l'histoire, / quand elles voient le roi assis sur son trône, / elles courent ensemble se jeter à ses pieds / tandis que Chantecler s'agenouille / et lui baigne les pieds de ses larmes.

Le traducteur a opté ici pour une participiale à l'ouverture et le présent dans le fragment entier, renonçant en quelque sorte à marquer le caractère séquentiel du récit.

De telles observations peuvent aider les étudiants à répérer les différences d'emploi des temps en ancien français et en français moderne. Une deuxième question qui se pose est de choisir une solution convenable pour la traduction en polonais. Cette dernière peut tirer parti de la liberté relative de l'emploi des temps

'Le Roman de Renart, éd. ct trad. en français mod. par Jean Dufournet et Andréc Mélinc, Flam. marion, Paris 1985, 2 vol. La traduction en français moderne qui suit csı de J. Dufournet et A. Méline.

${ }^{2}$ Ici, le récit se met au présent.

${ }^{3}$ L'incise est au présent. 
en ancien français, mais en même temps elle doit respecter les valeurs aspectuelles du texte original, ce dont les étudiants ne se rendent en général pas compte, n'ayant qu'une connaissance à peine rudimentaire de la grammaire polonaise. Reprenons l'exemple 1: la "toile de fond», c'est-à dire la défense de la paix par le roi (l'imparfait dans la version originale), peut s'exprimer en polonais au présent (toujours imperfectif, comme on sait):

Izengryn, shyszqc jak stanowczo ${ }^{4}$

król w państwie swym pokoju broni ${ }^{5}$,

tak się zawstydzil, tak się zmieszal ${ }^{6}$,

że odpowiedzieć nic nie zdołał.

Między dwa stołki wszedł po cichu

i usiadł, podkuliwszy ogon?

Une telle alternance aspectuelle est assez caractéristique pour la narration en polonais; mais pour qu'on l'applique, il faut tout simplement enseigner son utilisation aux étudiants, car d'eux-mêmes ils ne sont que rarement capables d'opérer un choix convenable.

Le deuxième exemple montre un autre cas de figure: aussi bien dans la version originale que dans la traduction, l'emploi du présent dans les deux derniers vers a une valeur dynamisante. Dans ce cas précis, il est possible de garder la même alternance aspectuelle et temporelle en polonais:

A potem, jak opowieść glosi ${ }^{8}$, kiedy już kury się ocknęly', zbliżyly się do lwiego tronu i do nóg władcy się rzuciły. Wraz z nimi klęka kogut Kantor ${ }^{10}$ i stopy pańskie skrapia łzami.

\section{L'ARGUMENTATION À DÉTECTER}

Il est notoire que l'ancien français use beaucoup la juxtaposition et la coordination qui parfois cachent des relations argumentatives (cause, justification, opposition, etc.) et opacifient les relations temporelles entre les événements. Les

\footnotetext{
${ }^{4}$ Une participiale de simultanćité.

${ }^{5}$ Le récit est au présent.

${ }^{6}$ A partir d'ici, le passé perfectif.

'Toutes les traductions en polonais: T. Giermak-Zieliniska, pour une anthologie de la littérature satirique médiévale, en préparation.

${ }^{8}$ L'incise au présent.

${ }^{9}$ A partir d'ici, le passé perfectif.

${ }^{10}$ Retour à la narration au présent.
} 
traductions en français moderne montrent différentes manières d'interpréter de telles phrases complexes. Parfois il existe plusieurs traductions d'un même ouvrage. On peut donc proposer aux étudiants de confronter l'argumentation de l'original et de la traduction (des traductions).

Voici quelques exemples où la juxtaposition cache une argumentation oppositive, cette dernière pouvant être inférée au niveau des connotations généralement liées au contenu de certains lexèmes:

Exemple 3 (Eliduc, 969-974) ${ }^{11}$

En la paumeisun la trovot:

Ne reveneit ne suspirot.

De ceo li semblot grant merveille

K'il la veeit blanche et vermeille:

Unkes la kolur ne perdi,

Fors un petit que ele enpali.

Traduction: Il la retrouve toujours sans connaissance, elle ne revenait pas à elle et ne respirait pas; mais ce qui lui sembla bizarre, c'est de la voir blanche et vermeille, elle n'a pas perdu ses couleurs, si ce n'est qu'elle a un peu pâli.

Souvent on peut se méprendre sur la valeur de «que», généralement associé avec la subordination, qui pourtant en ancien français peut revêtir la valeur d'un connecteur causal:

Exemple 4 (Boivin de Provins, 17-19) ${ }^{12}$

Une borse grant acheta

Douze deniers dedenz mis a,

Que il n'avoit ne plus ne mains ...

Traduction: Il acheta une grande bourse / y fourra dedans douze deniers / car il n'avait ni plus ni moins ...

Le connecteur car est indispensable dans la phrase moderne, puisqu'il explicite le rapport causal représenté par le que du texte ancien. De telles équivalences sémantiques ne posent pas beaucoup de problèmes, étant donné qu'elles sont à la portée de tout lecteur attentif d'un bon dictionnaire d'ancien français.

Il existe cependant de nombreux cas de structures argumentatives beaucoup plus complexes. Dans l'exemple 5 ci-dessous, il y a d'abord l'assertion concernant la sécurité conditionnelle du Renart («si Dex li oüst porveu») avec une justification introduite par $q^{\prime}$ («que»), donc formellement par une subordonnée, qui pourtant a la valeur d'une coordonnée introduite par «car». («car le roi, en dépit des mécontents,

" Marie de France, Lais, éd. bilingue par Alexandre Micha, Flammarion, Paris 1994. La traduction en français moderne qui suit est d'A. Micha.

${ }^{12}$ Fablianx, Textes traduits el présentés par Rosanna Bruscgan, coll. Bibliothèque médiévale, Editions 10/18, Paris, 1994. La traduction en français moderne qui suit est de R. Brusegan. 
a imposé la réconciliation en sorte que la guerre entre Renart et Isengrin aurait déjà pris fin si...»). La fin éventuelle (souhaitée, attendue) de la guerre est donc exprimée par un conditionnel contrefactuel. La troisième partie de la période apporte un retournement de la situation, exprimé de nouveau par un tour contrefactuel «Se ne fust Chantecler et Pinte» suivi de l'explication introduite par une relative «qui a la cort venoit soi quinte devant le roi de Renart pleindre».

Voici comment se présente le fragment entier:

Exemple 5 (Renart, branche 1, 274-281):

$\begin{array}{ll}\text { (assertion) } & \text { Or est Renart bien avenu } \\ \text { (condition contrefactuelle 1) } & \text { Si Dex li oüst porveü, } \\ \text { (justification) } & \text { Q'en tel point avoit pris li rois } \\ & \text { L'acorde, maugré les yrois, } \\ & \text { Que ja preïst la guerre fin } \\ & \text { Entre Renart et Ysengrin } \\ \text { (condition contrefactuelle 2) } & \text { Se ne fust Chantecler et Pinte } \\ \text { (explication) } & \text { Qui a la cort venoit soi qinte } \\ & \text { Devant lo roi de Renart pleindre. }\end{array}$

Traduction: Voilà donc une bonne nouvelle pour Renart / si sculement Dieu l'avait voulu. / Car le roi, en dépit des mécontents / a imposé la réconciliation / en sorte que la guerre entre Renart et Isengrin / aurait déjà pris fin / s'il n'y avait pas Chantecler et Pinte / qui étaient venus à la cour à cinq / pour porter plainte contre Renart devant le roi.

Dans la traduction polonaise l'assertion initiale au présent doit être remplacée par une clause hypothétique, sous peine de rendre le texte incompréhensible.

(hypothétique) Już Renart mógłby być bezpieczny, gdyby Bóg był po jego stronie.

(assertion) Tak to zakończył król obrady, ogłosil pokój mimo gniewów

(hypothétique) i już by się skończyła wojna lisa Renarta z Izengrynem, gdyby nic kogut Kantor, który samopięt z kurką Pstrą przybywa i chce na lisa złożyć skargę.

\section{LES CHOIX STYLISTIQUES}

On a beau donner aux étudiants le conseil d'adapter, dans la mesure du possible, le registre du texte traduit à l'original. Par manque d'expérience, mais aussi par manque d'habitude de lecture qui se perd, les étudiants ont souvent du mal à mettre ce principe en pratique. Souvent ils sont tentés par la solution la plus facile 
mais la moins bonne: ils restituent simplement le sens en choisissant un terme d'usage courant qui leur est familier. Sans leur suggérer l'option archaïsante, je les sensibilise à l'abus des termes trop récents ou trop courants. On fait de rapides exercices de mémoire afin de se rappeler des mots devenus rares, encore que non ressentis comme complètement dépassés: skibka chleba pour morceau de pain, trzewik pour soulier, osetka masta pour motte de beurre, etc. De même, je leur conseille de se renseigner sur les détails matériels de la vie d'autrefois, tels par exemple que l'équipement du guerrier et de son cheval, les noms des danses et des instruments anciens, etc. Un problème non moins important c'est l'usage social: comment traduire, sans tomber dans l'artificiel ou le ridicule, les anciennes tournures de politesse, d'appel, de refus, d'imprécation, etc. et, en fin de compte, des obscenités. Tout cela fait l'objet de discussion, en marge d'une traduction improvisée ensemble par tout le groupe, avant que les étudiants ne présentent leur travaux individuels.

La traduction des noms propres apporte encore de nouvelles difficultés. Je me bornerai ici à mentionner ceux occasionnés par les noms des protagonistes du récit. On y trouvera, grosso modo, deux sortes de noms: noms de baptême (dont plusieurs caractéristiques uniquement pour l'ancien français) et noms propres descriptifs.

Pour ce qui est du premier cas, le problème consiste à adapter phonétiquement au polonais des noms comme Robin et Marion ou Aucassin et Nicolette. Pour Robin et Marion, (titre d'un «jeu» d'Adam de la Halle), les diminutifs équivalents en polonais modeme seraient tout simplement ridicules (Robercik, Marysia), alors la démarche à suivre serait plutôt d'adapter phonétiquement les formes françaises: Robin ou Robinek, Marynka. Pour le deuxième couple de personnages, la solution adoptée par la traductrice ${ }^{13}$ est Alkasyn et Nikoleta, procédé où la polonisation passe par une reconstruction de la forme d'origine du nom masculin.

Les noms propres descriptifs ont un grand rôle à jouer, parce qu'ils constituent une partie importante du sens du texte entier. Tel est par exemple, le rôle des noms propres allégoriques dans Le Roman de la rose.

Contrairement à ce qui est caractéristique pour la métaphore (qui en général transfère un terme concret vers le domaine de l'abstrait), l'allégorie représente des propriétés abstraites personnifiées, le mouvement va donc de l'abstrait vers le concret (exemples: Dangiers, Contrainte Abstinence, Bel Accueil, Déduit, dame Raison, sans parler de cas plus simples: Doux Regard, Doux Penser, etc.). Le traducteur est confronté à deux problèmes: du côté du signifié, il doit préserver dans la langue d'arrivée les principales connotations du personnage allégorique, en sachant diversifier, par exemple, l'Envie et la Jalousie. Pour ce qui est de la forme du signifiant, sa tâche est de choisir un correspondant approprié par le genre grammatical au genre «naturel» du personnage allégorique, mais aussi d'éviter soigneusement des termes qui auraient eu un effet comique déplacé.

${ }^{13}$ Rzecz o Alkasynie i Nikolecie, trad. par Anna Ludwika Czerny, PIW, Warszawa 1962. 
Dans Le Roman de Renart on a affaire à des personnages humains couverts d'une peau de bête. Leurs noms ont des origines diverses et cela n'est pas sans conséquence pour la traduction. Il y a donc de "vrais» noms de baptême comme Renart (frq. Reginhart, Reinhart), des noms hérités d'une tradition littéraire comme Isengrin (Ysengrimus, titre de l'œuvre en latin de Nivart, $\mathrm{XII}^{e} \mathrm{~s}$.), des noms génériques accompagnés d'un épithète (sire Noble li Lions) et enfin des noms descriptifs à proprement parler: Chantecler, Pinte, Tardif le limaçon, Brichemer le cerf, Petit Porchaz le furet, l'ours Brun, des noms onomatopées: Frobert le grillon, enfin Percehaie (imitation comique de Perceval), etc.

L'inventaire de difficultés comprendra plusieurs points, tels que la structure grammaticale du nom, l'accord ou le conflit entre le genre «naturel» du personnage et le genre grammatical, enfins des connotations valorisantes (conflictuelles ou non entre la langue de départ et la langue d'arrivée). La solution du problème dépasse les seules compétences langagières, nécessitant un support des connaissances encyclopédiques, historiques, etc.

Ainsi donc nous sommes en plein dans le domaine des compétences cognitives ou, pour employer un terme courant, dans celui de la culture générale et de l'érudition. Le rôle de l'enseignant ici n'est évidemment pas d'enseigner la culture générale, comme on enseigne l'usage d'un mot ou d'une expression. Dans cette voie, l'étudiant doit s'engager seul, aidé et conseillé par son maître, mais c'est lui, et non son professeur, qui doit découvrir certaines choses, comme par exemple l'origine mythologique ou biblique des noms rencontrés dans le texte, leur forme dans la langue d'arrivée, confirmée par les dictionnaires ou parfois, seulement, par l'usage dans un texte littéraire polonais (qu'il faut savoir trouver) ou des traductions précédentes. L'érudition s'acquiert évidemment par des années de lecture et de réflexion, mais c'est justement à cause de cela qu'il faut y initier le futur traducteur avec un même soin que pour la justesse de l'expression.

Si par les compétences de traduction on entend aussi bien un savoir-faire linguistique qu'une part d'érudition non négligeable, ma profonde conviction est que les textes littéraires de l'ancien français sont un excellent matériau pour former des traducteurs sensibles à la totalité des enjeux que représente l'entreprise osée de traduire un texte littéraire. 